\title{
Un estudio comparativo entre los dispositivos T 200 DE COBRE Y ASA D DE LIPPES en mujeres en período de intervalo
}

\author{
Dres.: César Guzmán, M.D. ${ }^{1}$, Augusto Martínez, M.D. ${ }^{1}$ \\ Miguel Gutiérrez, M.D. ${ }^{1}$, Katherine S. Hilton, B.A. ${ }^{2}$
}

\section{INTRODUCCION}

La T 200 de Cobre y el Asa de Lippes son dos dispositivos intrauterinos (DIUs) que se han sometido a numerosas pruebas para determinar su seguridad y eficacia. En un estudio de control a doble ciego de los dos dispositivos, llevado a cabo en los Estados Unidos por Tietze y Lewit, que duró un año, se observó una tasa de fracasos de 2.2 con el T 200 de Cobre y de 3.0 con el Asa D de Lippes (1). En otra prueba similar en la que participaron mujeres en el período de intervalo, las tasas de fracaso variaron entre cero y 3.8 por cada cien mujeres, a los doce meses de la inserción (2).

El estudio que se describe a continuación es uno de los varios estudios que Family Health International ha llevado a cabo para comparar la seguridad $y$ eficacia de estos dispositivos intrauterinos, colocados a mujeres en el período de intervalo. En general, los dos dispositivos dieron resultados similares en

1. Centro Médico Carmen de la Legua, Lima, Perú.

2. Family Health International, Research Triangle Park, NC, USA. cuanto a complicaciones, quejas y descontinuación durante el período de seguimiento. Las tasas de continuación cumulativas de seis meses de la tabla de vida, fueron 96.6 para el T 200 de cobre y 91.4 para el Asa D de Lippes.

\section{MATERIAL Y METODOS}

\section{Dispositivos}

El Asa D de Lippes tiene la forma de una doble "S" y está hecha de polietileno impregnado con sulfato de bario. Este dispositivo es uno de los primeros DIUs modernos, $y$ el primero que incluyó el sulfato de bario en su composición.

La T 200 de Cobre es un dispositivo en forma de " $T$ ", hecho de polietileno y con un alambre de cobre enrollado alrededor del tallo vertical. El área total de cobre expuesto es de $200 \mathrm{~mm}^{2}$. Debido a que la T 200 de Cobre fue el primer dispositivo con cobre, que se sometió a pruebas en amplia escala, se le considera como la medida estándar para la evaluación de otros dispositivos intrauterinos medicados (3). Se supone que la presencia del cobre ayuda a prevenir el embarazo. 


\section{Metodología}

Desde marzo a fines de noviembre de 1982, un grupo de mujeres participó en una prueba experimental en el Centro Médico Carmen de la Legua de Lima, Perú. Se asignaron, al azar, 77 T 200 de Cobre y 64 Asas de Lippes a mujeres en el periodo de intervalo (42 días o más, después del parto o terminación del embarazo). Hay una diferencia en el número de mujeres en cada grupo que se debe a que el plan de distribución de DIUs al azar se hizo para un total de 200 mujeres; sin embargo, al momento de hacer el estudio se admitieron sólo 141 mujeres, por lo que el número de participantes en cada grupo no se pudo equiparar. Los dispositivos se colocaron con insertadores estándar. Las visitas de seguimiento para las mujeres se fijaron a los meses 1,3 y 6 después de la inserción. Todos los datos se recopilaron en formularios estandarizados, diseñados para obtener información médica y sociodemográfica así como información sobre cualquier trastorno durante el período de seguimiento.

\section{LOS RESULtAdDOS}

Los dos grupos de mujeres eran similares en cuanto a características sociodemográficas. La edad promedio entre las que recibieron la T 200 de Cobre era 27.3 años y 26.8 años entre las que recibieron el Asa D de Lippes. El número promedio de nacimientos vivos fue de 2.3 para la T 200 de Cobre y 2.7 para el Asa D de Lippes.

En el Cuadro No. 1 se presentan los niveles de complicaciones y quejas durante la inserción y durante el seguimiento. El único problema indicado durante la inserción fue dolor pélvico. En el grupo de la T 200 de Cobre, 20 mujeres $(26.0 \%$ ) experimentaron dolor pél. vico ligero o moderado en el momento de la inserción, mientras que en el grupo del Asa D de Lippes, 43 mujeres (67.2\%) indicaron lo mismo. Esta diferencia fue estadísticamente significativa $(p \leqslant 0.05)$. Una mujer a la que se le colocó un Asa $D$ de Lippes dijo sentir dolor severo durante la inserción.

Se obtuvieron datos sobre las complicaciones y las quejas en las visitas de seguimiento que se programaron a los meses 1, 3, 6 y después de la inserción. En cada grupo se citó la menorragia como la queja principal con relación a sangrado y dolor después de la inserción. El Cuadro No. 1 indica en detalle otras quejas de sangrado o dolor.

\section{Cuadro No. 1}

COMPLICACIONES Y QUEJAS DURANTE LA INSERCION Y EL SEGUIMIENTO*

\begin{tabular}{|c|c|c|c|c|}
\hline \multirow[b]{2}{*}{ Evento } & \multicolumn{2}{|c|}{$\begin{array}{c}T 220 \text { de Cobre } \\
(N=77)\end{array}$} & \multicolumn{2}{|c|}{$\begin{array}{c}\text { Asa D de Lippes } \\
\qquad(N=64)\end{array}$} \\
\hline & No. & $\frac{8}{8}$ & No. & 8 \\
\hline \multicolumn{5}{|l|}{$\begin{array}{l}\text { Complicaciones y quejas du- } \\
\text { rante la inserción }\end{array}$} \\
\hline \multicolumn{5}{|l|}{ Dolor Pélvico ** } \\
\hline Ligero & 14 & 18.2 & 21 & 32.8 \\
\hline Moderado & 6 & 7.8 & 22 & 34.4 \\
\hline Severo & 0 & 0.0 & 1 & 1.6 \\
\hline \multicolumn{5}{|l|}{$\begin{array}{l}\text { Complicaciones y quejas du- } \\
\text { rante el seguimiento }\end{array}$} \\
\hline \multicolumn{5}{|c|}{ Queja principal de sangrado/dolor } \\
\hline Menorragia & 16 & 22.5 & 13 & 22.0 \\
\hline \multicolumn{5}{|l|}{ Intermenstrual } \\
\hline Sangrado & 4 & 5.6 & 12 & 20.3 \\
\hline Manchado & 7 & 9.9 & 8 & 13.6 \\
\hline Dolor & 4 & 5.6 & 3 & 5.1 \\
\hline Dismenorrea & 5 & 7.0 & 4 & 6.8 \\
\hline Inflamaciones pélvicas*** & 6 & 8.0 & 0 & 0.0 \\
\hline Dispareunia & 3 & 4.2 & 0 & 0.0 \\
\hline Condiloma & 1 & 1.6 & 0 & 0.0 \\
\hline -. Puede haberse notificad & o & $/ \mathrm{dol}$ & ma & \\
\hline $\begin{array}{c}\cdots \quad p \leqslant 0.05 \\
\cdots \quad p \leqslant 0.02\end{array}$ & & & & \\
\hline
\end{tabular}

Se diagnosticaron inflamaciones pélvicas en seis mujeres que estaban usando la T 200 de Cobre, en el período entre uno y doce meses después de la inserción. Una mujer tuvo inflamación pélvica 
limitada a la matriz, cuatro tuvieron inflamación pélvica hasta los anexos y una mujer tuvo inflamación pélvica de la matriz y anexos. Ninguna de las mujeres en el grupo del Asa $D$ de Lippes dijo haber tenido inflamación pélvica. La diferencia en la presencia de inflamaciones pélvicas entre los dos grupos fue estadísticamente significativa $(p \leqslant 0.02)$. Se observaron tres casos de dispareunia en las mujeres del grupo de la T 20 o de Cobre. A una mujer que estaba usando la T 200 de Cobre se le diagnósticó condiloma de la vulva.

Las tasas cumulativas de la tabla de vida se presentan en el Cuadro No. 2. Hubieron tres embarazos en el grupo del Asa D de Lippes, produciendo una tasa de 3.8 a los seis meses. No hubieron embarazos en el grupo de la T 200 de

Cuadro No. 2

TASAS CUMULATIVAS DE LA TABLA DE VIDA POR 100 MUJERES

\begin{tabular}{|c|c|c|}
\hline Tipo de Conclusión y Período & $\begin{array}{c}T \text { de Cobre } \\
(N=77)\end{array}$ & $\begin{array}{l}\text { Asa D de Lippes } \\
\qquad(N=64)\end{array}$ \\
\hline \multicolumn{3}{|l|}{ Embarazo accidental } \\
\hline 1 mes & $0.0 \pm 0.0$ & $0.0 \pm 0.0$ \\
\hline 3 meses & $0.0 \pm 0.0$ & $1.8 \pm 1.8$ \\
\hline 6 meses & $0.0 \pm 0.0$ & $3.8 \pm 2.6$ \\
\hline \multicolumn{3}{|l|}{ Expulsión/cambio de lugar } \\
\hline 1 mes & $1.4 \pm 1.3$ & $3.3 \pm 2.3$ \\
\hline 3 meses & $1.4 \pm 1.3$ & $3.3 \pm 2.3$ \\
\hline 6 meses & $3.4 \pm 2.4$ & $3.3 . \pm 2.3$ \\
\hline \multicolumn{3}{|l|}{ Sangrado/dolor } \\
\hline 1 mes & $0.0 \pm 0.0$ & $0.0 \pm 0.0$ \\
\hline 3 meses & $0.0 \pm 0.0$ & $1.8 \pm 1.8$ \\
\hline 6 meses & $0.0 \pm 0.0$ & $1.8 \pm 1.8$ \\
\hline \multicolumn{3}{|l|}{ Tasa de continuación } \\
\hline 1 mes & 98.6 & 96.7 \\
\hline 3 meses & 98.6 & 93.3 \\
\hline 6 meses & 96.6 & 91.4 \\
\hline \multicolumn{3}{|l|}{ Tasa de seguimiento* } \\
\hline 1 mes & 92.2 & 92.2 \\
\hline 3 moses & 86.8 & 88.3 \\
\hline 6 meses & 69.7 & 76.3 \\
\hline
\end{tabular}

La tasa de seguimiento se define como el porcentaje de mujeres a las que no 5 ha retirado del estudio y que regresan para los exámenes de control
Cobre. Las tasas de expulsión fueron similares para los dos grupos, 3.4 para las que usaban la T 200 de Cobre y 3.3 para las que usaban el Asa D de Lippes. La mayoría de las expulsiones se produjeron durante el primer mes después de la inserción. La tasa de extracción debido a sangrado o clolor fue de 1.8 en el grupo del Asa $D$ de Lippes. No hubieron extracciones por sangrado o dolor en el grupo de la T 200 de Cobre. La tasa de continuación para las mujeres que usaban la T 200 de Cobre fue de 96.6, con una tasa de seguimiento de 69.7 a los seis meses. La tasa de continuación para el grupo del Asa D de Lippes fue de 91.4 y la tasa de seguimiento fue de 76.3 a los seis meses.

\section{DISCUSION}

Los dispositivos intrauterinos ofrecen un método anticonceptivo reversible que requiere poco esfuerzo por parte de las usuarias, particularmente si se les compara con los anticonceptivos orales - los métodos de barrera. Se han hecho DIUs más pequeños para mejorarlos y para reducir los niveles de sangrado y dolor asociados con los dispositivos más grandes. El cobre se ha añadido al tallo del DIU debido a su potencial anticonceptivo (4).

Family Health International ha llevado a cabo varios estudios comparativos con los dispositivos T 200 de Cobre y Asa $D$ de Lippes para determinar su seguridad y eficacia cuando son colocados en el período de intervalo. Uno de estos estudios se llevó a cabo en Lima, Perú. Las diferencias en los resultados observados en los dos grupos fueron evidentes. Es aparente que un mayor número de mujeres sintieron dolor durante la inserción en el grupo del Asa D de Lippes, que es posible atribuir al tamaño más grande de este dispositivo. Otro resultado indica que se da un nú- 
mero mayor de inflamaciones pélvicas en el grupo que usa la T 200 de Cobre y la diferencia es estadísticamente significativa $(p \leqslant 0.02)$. No hay evidencia clara de las razones por las que se dio este hecho.

\section{Reconocimiento}

Family Health International dio apoyo parcial para este estudio con fondos proporcionados por la Agencia Internacional para el Desarrollo (AID).

\section{RESUMEN}

En un estudio llevado a cabo en el Centro Médico Carmen de la Legua, en Lima, Perú, se compararon los dispositivos T 200 de Cobre y Asa D de Lippes, colocados a mujeres en el periodo de

\section{REFERENCIAS}

1. TIETZE C., LEWIT S., Comparison of the copper $T$ and loop D: A research report. Studies in Family Planning, 1976; 3 (11): 277. 278.

2. John Hopkins University. Population Reports,1982; Series B, Number 4 B 103.

3. TATUM H., Clinical aspects of intrauterine contraception: Circumspection 1976. Ferti- intervalo. Los dispositivos se distribuyeron al azar y se insertaron con insertadores estándar. La actuación de los dos dispositivos fue similar. Sin embargo, se observó una diferencia estadísticamente significativa $(p<0.02$ ) en cuanto a la presencia de inflamaciones pélvicas asociadas al uso de los dispositivos, en los dos grupos. Se produjeron seis casos de inflamaciones pélvicas en el grupo que usó la T 200 de Cobre, mientras que no se observó ningún caso en el grupo que usó el Asa D de Lippes. Se confirmaron tres embarazos en el grupo que usó el Asa D de Lippes. Las tasas de continuación a los seis meses, fueron de 96.6 para las mujeres que estaban usando el $\mathrm{T}$ de Cobre y 91.4 para las que estaban usando el Asa D de Lippes.

TERMINOS CLAVES: Dispositivo intrauterino (DIU), T 200 de Cobre, Asa D de Lippes, Inserción intervalo.

lity and Sterility, 1977; Vol. 28, No. 1: 3-28.

4. ZIPPER J., TATUM H., PASTENE L., MEDEL M., RIVERA M., Metallic Copper as an intrauterine contraceptive adjunct to the " $\mathrm{T}$ " device. American Journal of Obstetrics and Gynecology 1969; Vol. 105, No. 8:1274-1278. 